\title{
CT Optimization for Diagnosis of Some Acute Abdomen Cases
}

\author{
Saddig D. Jastaniah ${ }^{1}$, Alamin M. Salih', Khalid Gh. Alsafi2,3, Hamad Elniel H. Eltyib1, \\ Sarah Hagi ${ }^{2,3}$, Maway A. Khafaji ${ }^{2,3}$, Hanan Y. Abbas ${ }^{1}$, Mohammad Alshihri ${ }^{1}$ \\ ${ }^{1}$ Department of Diagnostic Radiology, Faculty of Applied Medical Sciences, King Abdulaziz University, Jeddah, \\ Kingdom of Saudi Arabia \\ ${ }^{2}$ Radiology Department, King Abdulaziz University Hospital, Jeddah, Kingdom of Saudi Arabia \\ ${ }^{3}$ Department of Radiology, School of Medicine, King Abdulaziz University, Jeddah, Kingdom of Saudi Arabia \\ Email: sjastaniah@kau.edu.sa
}

Received 11 December 2014; accepted 29 May 2015; published 1 June 2015

Copyright (C) 2015 by authors and Scientific Research Publishing Inc.

This work is licensed under the Creative Commons Attribution International License (CC BY).

http://creativecommons.org/licenses/by/4.0/

(c) (i) Open Access

\section{Abstract}

The acute abdomen is one of the most frequent causes for presentation to the emergency department. Imaging plays an important role for an accurate diagnosis, which in turn diminishes morbidity and mortality. The aim of this study was to demonstrate optimum CT aspects and emphasize on the important features of CT for those patients presenting with an acute abdominal pain at the Emergency Department both in general and in a number of selected conditions (appendicitis, small-bowel obstruction, acute pancreatitis, and diverticulitis). The reported data by this study are based on the author working experience, which forms a continuous protocol adjustment process. The present study provides evidence that CT would result in definite diagnosis of patients with abdominal pain in terms of the detection of some urgent conditions.

\section{Keywords}

\section{CT, Acute Abdominal Pain, Appendicitis, Small-Bowel Obstruction}

\section{Introduction}

The acute abdomen may be defined generally as an intra-abdominal process causing severe pain and often requiring medical or surgical intervention. The acute abdomen is a frequent entity at the Emergency Department, the acute abdomen may be life threatening and so rapid diagnosis of patients presenting with acute abdominal

\footnotetext{
*Corresponding author.
}

How to cite this paper: Jastaniah, S.D., Salih, A.M., Alsafi, K.Gh., Eltyib, H.E.H., Hagi, S., Khafaji, M.A., Abbas, H.Y. and Alshihri, M. (2015) CT Optimization for Diagnosis of Some Acute Abdomen Cases. Advances in Computed Tomography, 4, 19-26. http://dx.doi.org/10.4236/act.2015.42003 
pain is required [1].

Diagnostic work-up with imaging can consist of plain X-ray, ultrasonography (US), computed tomography (CT) and even diagnostic laparoscopy. During the last decade, a trend towards increased use of computed tomography in patients with abdominal pain can be seen [2] [3]. In general, acute abdominal pain is responsible for about $10 \%$ of total number of patients visiting the emergency. Quick and precise diagnosis for the urgent cases is usually vital for treatment [4].

Medical imaging is commonly used for the diagnosis of most acute abdomenpain [5]. Abdomen and pelvis are reviewed by CT scan. Specifically, in cases of acute abdomen complain [6].

The sensitivity, specificity, and accuracy of unenhanced helical CT are 96.0\%, 95.1\%, and 95.6\%, respectively. But, radiograph series of acute abdominal was reported to have an overall sensitivity, specificity, and accuracy of $30.0 \%, 87.8 \%$, and $56 \%$, respectively [7].

The aim of this study was to demonstrate optimum CT aspects and emphasize on the important features of CT for those patients presenting with an acute abdominal pain at the Emergency Department both in general and in a number of selected cases as described in the results section. Similar studies by the authors were previously reported [8].

\section{Materials and Methods}

After the approval of Human Ethics of King Abdulaziz University, this study retrospectively reviewed electronically available records for patients admitted to the Emergency Department. All patients examined on a multislice CT scanner (64 slice Siemens somatom definition dual source), and the contrast administrated using automatic power injector, there is no inclusion criteria for the selection of patients, but all patient who came to examine CT abdomen with acute abdominal pain has been included. Different patient preparation and different CT protocols were conducted in order to know the nature of diseases that may lead to acute abdomen pain. Imaging protocols are dependent on the possible diagnosis, clinical adjustment, and radiographer's experience. Factors that should be adjusted to each patient includes: slice collimation and pitch; the use of oral or IV material; and limited-focus scan versus a complete abdominal examination. Summarized clinical findings, as in routine practice, will be provided. The CT scan was evaluates and records data in a similar way. The CT protocols used are described below.

\subsection{Pancreatitis Protocol}

The patient is prepared, he/she was asked to fast for several hours (NPO 4-6 hours), fresh serum creatinine and GFR are required, if IV contrast will be used during an examination. Water or low-attenuation oral contrast agents are preferred because dense contrast may obscure small stones, also to delineate the duodenal wall with possibility to perform CT angiography without beam hardening artifacts caused by bowel contrast. The protocol is described in Table 1.

\subsection{Abdomen and Pelvis/Small Bowel Obstruction}

The patient is prepared by fasting for several hours (NPO 4-6 hours), fresh serum creatinine and GFR are required, if the patient candidate for IV contrast. The protocol is listed in Table 2.

\subsection{Abdomen and Pelvic: Urolithiasis}

In Table 3, Urolithiasis (a renal stone) scanning protocol is described. There are some important points regarding abdomen and pelvic exam. If patient is able, scan the patient in the prone position. This is useful for differentiating between an ureterovesical junction (UVJ) stone and a passed stone. No patient preparation is required because of this is a non-contrast study, however, better patient hydration through the ingestion of water before the study can help to eliminate small hyper densities of the renal pyramids that can mimic stones. Thin slices allow identification of small stones that may be overlooked with thicker slices. The radiation dose should be kept minimum particularly to the gonads. It is important because many patients who have stone are young and may have repeated stone formation. Therefore, might undergo CT again several times in the future. Lower dose techniques can reduce the exposure but exposure can still be high if multiple examinations are obtained. 
Table 1. Pancreatitis protocol.

\begin{tabular}{cc}
\hline Indications & Pancreatitis \\
Patient position & Supine arms elevated above head \\
To program & From above diaphragm to pubic symphysis \\
Breathing breath & hold in inspiration (single breath hold) \\
Contrast & Oral: $1000 \mathrm{ml}$ water over $30 \mathrm{~min}, \mathrm{I.V}: 110 \mathrm{ml}$ at $3 \mathrm{ml} / \mathrm{s}$ \\
Timing for & Pancreatitis/arterial follow up $65 \mathrm{~s}$ and 120 delay \\
Technical parameters & Pitch: 1 Suggested collimation $64 \times 0.6 \mathrm{~mm}$, \\
Comments & Water as oral contrast is preferred (pancreatitis) \\
\hline
\end{tabular}

Table 2. Abdomen and pelvis/small bowel obstruction.

\begin{tabular}{|c|c|}
\hline Contrast protocol & Parameters \\
\hline Oral contrast & Positive, if possible/tolerable \\
\hline Rectal contrast & Usually not necessary \\
\hline IV contrast-iodine conc. (mgl/mL) & 300 \\
\hline Volume (mL) & $100-115$ \\
\hline Iodine dose (gI) & 46 \\
\hline Flow rate $(\mathrm{mL} / \mathrm{s})$ & $2.8-3.0$ \\
\hline Iodine dose rate (gI/s) & 1.2 \\
\hline Saline flush-volume (mL) & 50 \\
\hline Saline flush-flow rate $(\mathrm{mL} / \mathrm{s})$ & 3 \\
\hline Scan delay (s) & 70 or care bolus \\
\hline Delayed scans & Not necessary \\
\hline Scanning Protocol & Parameters \\
\hline Scout (to program) & Anterior-posterior \\
\hline Patient position & Supine \\
\hline Scan range & Diaphragm to symphysis \\
\hline Scan direction & Cephalocaudal \\
\hline Tube voltage (KVp) & 120 \\
\hline Effective mAs & 170 to 200; use CARE Dose 4D \\
\hline Gantry rotation time (s) & 0.5 \\
\hline Slice collimation $(\mathrm{mm})$ & $64 \times 0.6$ \\
\hline Pitch & 0.9 \\
\hline Table feed (mm/rotation) & 17.28 \\
\hline & Reconstruction parameters \\
\hline Slice width (mm) & 5 \\
\hline Axial slice width for 3D/MPR (mm) & 2 \\
\hline Recon. increment (mm) & 5 \\
\hline Axial recon. increment for 3D/MPR (mm) & 1 \\
\hline Special views & Coronal \\
\hline Recon. kernel & B20f/B30f \\
\hline Recon. field of view & Fit to patient \\
\hline
\end{tabular}

Positive oral contrast is given to patient if they can tolerate but usually not necessary: ${ }^{*}$ Obstructive patient have excessive of enteric fluid. *Surgeon may not prefer oral contrast prior surgery.-IV contrast portal venous phase occasionally dual phase of concern for closed lobe or ischemia-MPR is useful to identify the site, level and cause of obstruction, when axial is indeterminate. 
Table 3. Abdomen and pelvic: urolithiasis.

\begin{tabular}{cc}
\hline Scanning protocol & Acquisition parameters \\
Scout (topogram) & A-P \\
Patient position & Prone \\
Scan range & Top of kidneys to symphysis \\
Scan direction & Cephalocaudal \\
Tube voltage (kVp) & 100 or less \\
Effective mAs & 50 use CARE Dose 4D \\
Gantry rotation time (s) & 0.5 \\
Slice collimation (mm) & $64 \times 0.6$ \\
Pitch & 0.9 \\
Table feed (mm/rotation) & 17.28 \\
Reconstruction Parameters & Fit to patient \\
Slice width (mm) & 2 \\
Axial slice width for3D/MPR (mm) & 2 \\
Recon. Increment (mm) & 2 \\
Axial recon. increment for & \\
SD/MPR (mm) & 2 \\
Special views & \\
\hline
\end{tabular}

\section{Results}

\subsection{Acute Pancreatitis}

Edema of the pancreas and surrounding fat is clearly demonstrated in the CT findings of acute pancreatitis. The whole gland could be enlarged. By enhancement to the islands of viable tissue could become scattered throughout the gland as demonstrated in Figure 1.

\subsection{Diverticulitis}

Fever, pain, and leukocytosis are associated with Patients that have diverticulosis usually happening with left lower quadrant. Inflammation and abnormality in the pericolic fat is observed in the CT of diverticulitis. Phlegmon formation can occur in severe cases. The CT images of the inflamed diverticulum shown as a rounded paracolic outpouching in the center of parabolic inflammation Figure 2.

\subsection{Small Bowel Obstruction (SBO)}

CT findings of bowel obstruction are normally the delineation of a transition zone appearing between decompressed bowels and dilated as demonstrated in Figure 3. If there is no hernia, mass, or inflammatory thickening; adhesion is clearly the right diagnosis.

\subsection{Urolithiasis}

Non-contrasted CT calcified and non-calcified urinary stones were identified, along with the location and size of the stone from kidney to bladder. Secondary signs of obstructive uropathy, including hydronephrosis and ureteral ecstasies were noticed. On non-contrast CT, calcified urinary stones appear as opaque densities within the urinary tract to differentiate calcified and non-calcified urinary stones according to their appearance are not 


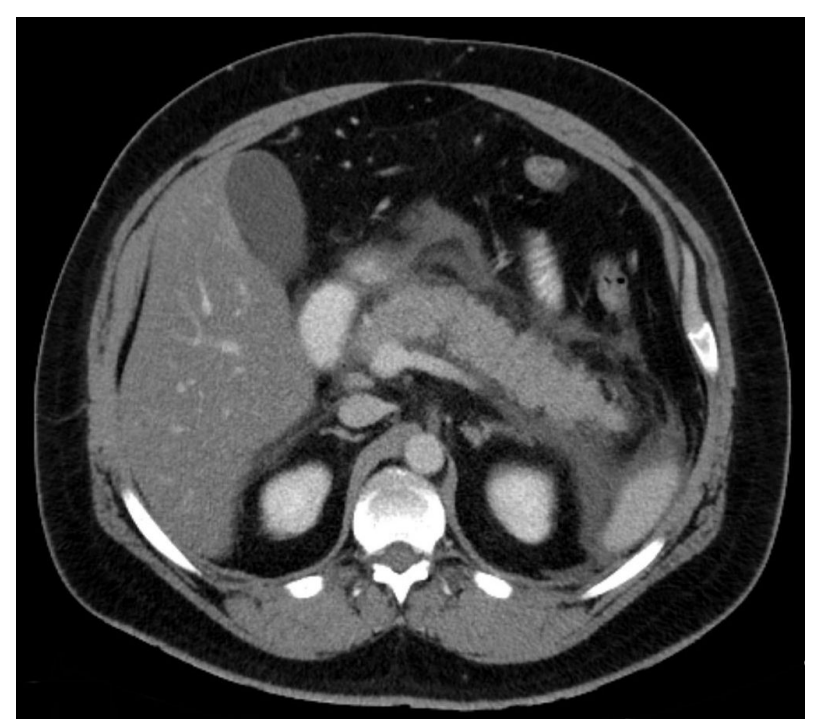

Figure 1. Acute pancreatitis.

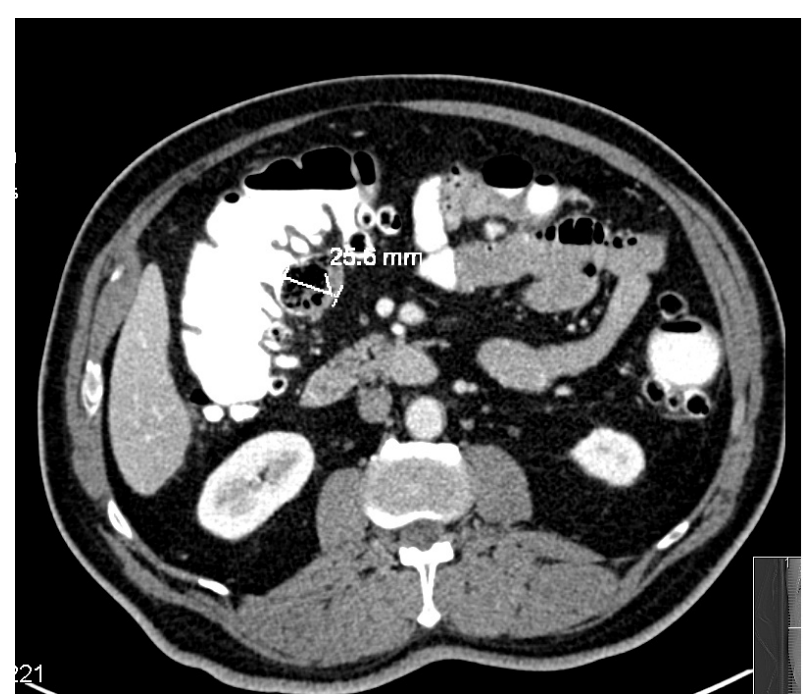

Figure 2. Diverticulosis showing abnormality.

possible. Degree of accuracy in interpreting a non-contrast CT in a case with urinary stone increases in accordance with the severity of urinary obstruction.

\section{Discussion}

Different patient preparation and different CT protocols were conducted in order to know the nature of diseases that may lead to acute abdomen pain. Imaging protocols are dependent on the possible diagnosis, clinical adjustment, and radiographer's experience [8] [9].

Both CT and ultrasound plays important roles in acute appendicitis diagnosis. CT is found to be more accurate thus was the preferred imaging modality [10] [11].

Small-bowel obstruction (SBO) is a common cause of acute abdomen. Scanning was performed from the diaphragm to the pubic symphysis with a CT scanner. The transverse plus coronal scans were more confident and diagnostic for the presence of SBO than for the transverse scans alone [12].

When the transition zone of the luminal contents is carefully inspected this will usually tell the obstruction reason [13] [14]. [15] proved that sub millimeter coronal reformations add to the confidence level of readers for the diagnosis of acute appendicitis. 


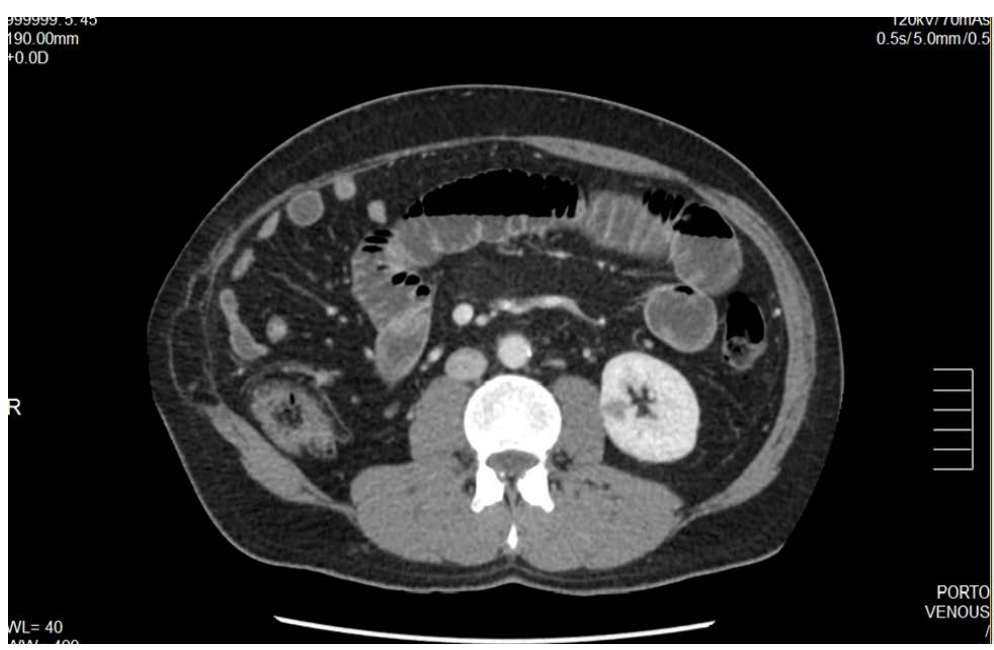

(a)

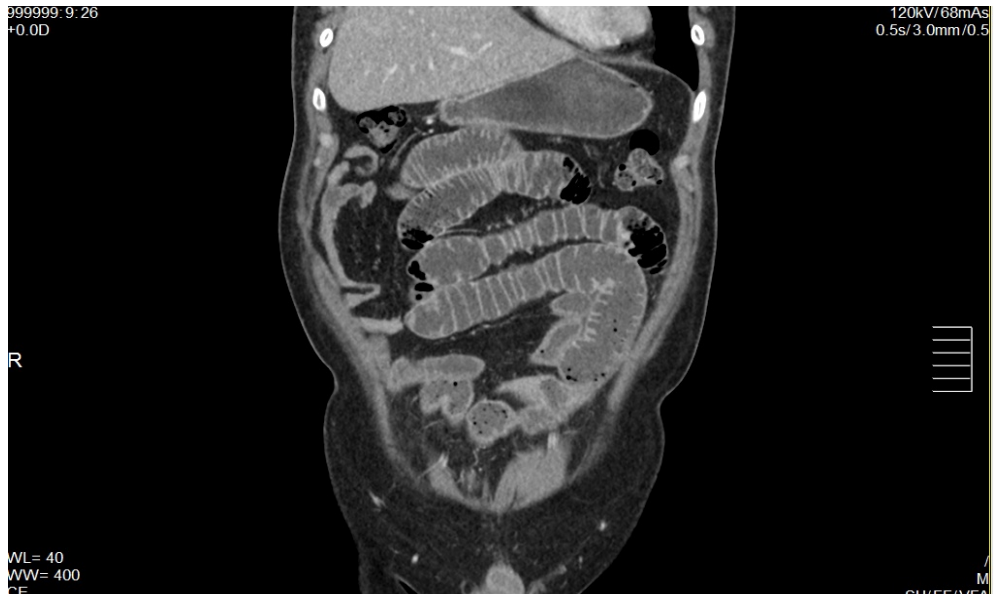

(b)

Figure 3. (a) Small bowel obstruction; axial cut; (b) Small bowel obstruction; coronal cut.

Acute colonic diverticulitis normally do not show any typical image features. Therefore, CT is will ensure suspected diagnosis by detecting any complication that might be present [16]. It has been suggested that the use of plain imaging should be excluded from the initial assessment of these patients [17].

Previous studies demonstrated that CT allowed diagnosis and determining the size, composition, and location of stones [18]. This was done by analyzing the correlation between dimensions of stone using CT scan assessment and plain X-ray of the kidneys, bladder and ureter. Other studies [19] [20] to evaluate calculi, it was possible to demonstrate a sensitivity of $97 \%$, specificity of $96 \%$, and accuracy of $97 \%$ in 60 patients. Identification of the number, size, and location of urinary stones and detection of hydronephrosis are easily made with CT.

SBO is a relatively common cause of acute abdominal pain. Combination of vomiting, increased bowel sounds, and a distended abdomen has a positive predictive value for SBO of 64\% [21]. The exact location or cause of SBO may not be diagnosed via plain abdominal imaging [22]. Instead, CT is capable to obtain obstruction causes. [23] [24] suggested that multiplanar reformations are helpful for the evaluation of SBO.

In our study, SBO was compared to the previously identified CT scout scans [25] [26]. These results corroborate recent reports [12]. Our results suggest that the coronal plane serves as a useful addition to the transverse plane in patients suspected of having SBO.

From radiation protection point of view, lower dose techniques can reduce the exposure but exposure can still be high if multiple examinations are obtained this can be Some sort of limitations for optimizing protocols implemented in CT investigations. 


\section{Conclusion}

The present study provides evidence that CT would result in definite diagnosis for detecting urgent conditions in patients with abdominal pain. Proper technique and protocol are essential for optimizing the CT examination and maximizing diagnostic accuracy. CT scanning has gained acceptance as the primary imaging techniques for the SBO, Urolithiasis and acute appendicitis.

\section{References}

[1] Trott, A.T. and Lucas, R.H. (1998) Acute Abdominal Pain. In: Rose, P., Ed., Emergency Medicine, 4th Edition, St. Louis, Mosby, 1888-1903.

[2] Weyant, M.J., Eachempati, S.R., Maluccio, M.A., Rivadeneira, D.E., Grobmyer, S.R., Hydo, L.J., et al. (2000) Interpretation of Computed Tomography Does Not Correlate with Laboratory or Pathologic Findings in Surgically Confirmed Acute Appendicitis. Surgery, 128, 145-152. http://dx.doi.org/10.1067/msy.2000.107422

[3] Lee, S.T., Walsh, A.J. and Ho, S.H. (2001) Computed Tomography and Ultrasonography Do Not Improve and May Delay the Diagnosis and Treatment of Acute Appendicitis. Archives of Surgery, 136, 556-562. http://dx.doi.org/10.1001/archsurg.136.5.556

[4] Martin, R.F. and Rossi, R.L. (1997) The Acute Abdomen: An Overview and Algorithms. Surgical Clinics of North America, 77, 1227-1243. http://dx.doi.org/10.1016/S0039-6109(05)70615-0

[5] Schurink, G.W, Bode, P.J., van Luijt, P.A. and van Vugt, A.B. (1997) The Value of Physical Examination in the Diagnosis of Patients with Blunt Abdominal Trauma: A Retrospective Study. Injury, 28, 261-265. http://dx.doi.org/10.1016/s0020-1383(97)00007-7

[6] Stoker, J., van Randen, A., Laméris, W. and Boermeester, M.A. (2009) Imaging Patients with Acute Abdominal Pain. Radiology, 253, 31-46. http://dx.doi.org/10.1148/radiol.2531090302

[7] MacKersie, A.B., Lane, M.J., Gerhardt, R.T., et al. (2005) Nontraumatic Acute Abdominal Pain: Unenhanced Helical CT Compared with Three-View Acute Abdominal Series. Radiology, 237, 114-122. http://dx.doi.org/10.1148/radiol.2371040066

[8] Jastaniah, S.D. and Salih, A.M. (2014) Helical Computed Tomography in Evaluation of Selected Cases of Acute Abdomen. Advances in Computed Tomography, 3, 31-38; Published Online September 2014 in SciRes.

[9] Mindelzun, R.E. and Jeffrey, R.B. (1999) The Acute Abdomen: Current CT Imaging Techniques. Seminars in ULTRASOUND CT and MRI, 20, 63-67. http://dx.doi.org/10.1016/S0887-2171(99)90037-9

[10] Cobben, L.P., Groot, I., Haans, L., Blickman, J.G. and Puylaert, J. (2004) MRI for Clinically Suspected Appendicitis during Pregnancy. American Journal of Roentgenology, 183, 671-675. http://dx.doi.org/10.2214/ajr.183.3.1830671

[11] Vu, L., Ambrose, D., Vos, P., Tiwari, P., Rosengarten, M. and Wiseman, S. (2009) Evaluation of MRI for the Diagnosis of Appendicitis during Pregnancy When Ultrasound Is Inconclusive. Journal of Surgical Research, 156, 145-149. http://dx.doi.org/10.1016/j.jss.2009.03.044

[12] Gore, R.M., Miller, F.H., Pereles, F.S., Yaghamai, V. and Berlin, J.W. (2000) Helical CT in the Evaluation of the Acute Abdomen. American Journal of Roentgenology, 174, 901-913. http://dx.doi.org/10.2214/ajr.174.4.1740901

[13] Maglinte, D.D., Heitkamp, D.E, Howard, T.J., Kelvin, F.M. and Lappas, J.C. (2003) Current Concepts in Imaging of Small Bowel Obstruction. Radiologic Clinics of North America, 41, 263-283. http://dx.doi.org/10.1016/S0033-8389(02)00114-8

[14] Maglinte, D.D., Kelvin, F.M., Sandrasegaran, K., et al. (2005) Radiology of Small Bowel Obstruction: Contemporary Approach and Controversies. Abdominal Imaging, 30, 160-178. http://dx.doi.org/10.1007/s00261-004-0211-6

[15] Paulson, E.K., Harris, J.P., Jaffe, T.A., Haugan, P.A. and Nelson, R.C. (2005) Coronal Reformations from Isotropic Voxels Using 16 Slice MDCT: Value in the Diagnosis of Acute Appendicitis. Radiology, 235, 879-885. http://dx.doi.org/10.1148/radiol.2353041231

[16] Ming, S.-C. (1999) Diverticular Disease of the Colon. In: Ming, S.-C. and Goldman, H., Eds., Pathology of the Gastrointestinal Tract, 2nd Edition, Williams \& Wilkins, Baltimore, 801-818.

[17] van Randen, A., Laméris, W., Luitse, J.S., et al. (2009) The Role of Plain Radiographs in Patients with Acute Abdominal Pain at the ED. American Journal of Emergency Medicine, 29, 582-589.

[18] Tisdale, B.E., Siemens, D.R., Lysack, J., Nolan, R.L. and Wilson, J.W. (2007) Correlation of CT Scan versus Plain Radiography for Measuring Urinary Stone Dimensions. The Canadian Journal of Urology, 14, 3489-3492.

[19] Liu,W., Esler, S.J., Kenny, B.J., Goh, R.H., Rainbow, A.J. and Stevenson, G.W. (2000) Low-Dose Nonenhanced Helical CT of Renal Colic: Assessment of Ureteric Stone Detection and Measurement of Effective Dose Equivalent. Radiology, 215, 51-54. http://dx.doi.org/10.1148/radiology.215.1.r00ap4051 
[20] Lin, W.C., Uppot, R.N., Li, C.S., Hahn, P.F. and Sahani, D.V. (2007) Value of Automated Coronal Reformations from 64-Section Multidetector Row Computerized Tomography in the Diagnosis of Urinary Stone Disease. The Journal of Urology, 178, 907-911. http://dx.doi.org/10.1016/j.juro.2007.05.042

[21] Bohner, H., Yang, Q., Franke, C., Verreet, P.R. and Ohmann, C. (1998) Simple Data from History and Physical Examination Help to Exclude Bowel Obstruction and to Avoid Radiographic Studies in Patients with Acute Abdominal Pain. European Journal of Surgery, 164, 777-784. http://dx.doi.org/10.1080/110241598750005435

[22] Frager, D., Medwid, S.W., Baer, J.W., Mollinelli, B. and Friedman, M. (1994) CT of Small Bowel Obstruction: Value in Establishing the Diagnosis and Determining the Degree and Cause. American Journal of Roentgenology, 162, 37-41. http://dx.doi.org/10.2214/ajr.162.1.8273686

[23] Caoili, E.M. and Paulson, E.K. (2000) CT of Small-Bowel Obstruction: Another Perspective Using Multiplanar Reformations. American Journal of Roentgenology, 174, 993-998. http://dx.doi.org/10.2214/ajr.174.4.1740993

[24] Furukawa, A., Yamasaki, M., Furuichi, K., et al. (2001) Helical CT in the Diagnosis of Small Bowel Obstruction. Radiographics, 21, 341-355.

[25] Maglinte, D.D., Reyes, B.L., Harmon, B.H., et al. (1996) Reliability and Role of Plain Film Radiography and CT in the Diagnosis of Small Bowel Obstruction. American Journal of Roentgenology, 167, 1451-1455. http://dx.doi.org/10.2214/ajr.167.6.8956576

[26] Lappas, J.C., Reyes, B.L. and Maglinte, D.D. (2001) Abdominal Radiography Findings in Small-Bowel Obstruction. American Journal of Roentgenology, 176, 167-174. http://dx.doi.org/10.2214/ajr.176.1.1760167 